\title{
A economia neoclássica: uma análise lakatosiana Da cheia do mainstream até sua implosão*
}

\author{
The Neoclassical economics: a Lakatosian analysis. \\ Fom full minstream until its implosion
}

ALAIN HERSCOVICI**

RESUMO: Este trabalho tem como objetivo definir, a partir de uma abordagem lakatosiana, o Programa de Pesquisa Científica (PPC) Neoclássico. Para tal fim, vou comparar este programa com aqueles oriundos da nova economia institucional (Coase e Williamson) e da nova economia da informação (Akerlof, Grossman e Stiglitz) e, usando a noção de implosão, vou destacar os seus limites. Depois de discutir o método de Lakatos e, baseando-se em sua abordagem, definirei o PPC neoclássico, e explicarei em que consiste a implosão do SRP neoclássico.

PALAVRAS-CHAVE: epistemologia econômica; economia neoclássica; critério de demarcação.

ABSTRACT: Neoclassical Economics: a Lakatosian approach. This paper aims to define, from a Lakatosian approach, the neoclassical scientific research program (SRP). For such purpose, I will compare this SRP with the ones of the new institutional economics (Coase and Williamson) and the New Information Economics (Akerlof, Grossman e Stiglitz) and, by using the notion of implosion, I will highlight its limits. After discussing Lakatos' method and drawing upon his approach to define the Neoclassical SRP, the paper defines and explain the Neoclassical SRP implosion.

KEYWORDS: economic epistemology; Neoclassical Economics; demarcation criterion. JEL Classification: B41; B2.

\footnotetext{
* O subtítulo faz alusão ao texto de Mário Possas intitulado "A cheia do mainstream” (1995). Agradeço os comentários de um parecerista anônimo; isto permitiu retificar certos erros, reestruturar e melhorar assim substancialmente este trabalho. Obviamente, continuo sendo inteiramente responsável pelo conteúdo deste artigo.

* Professor Titular, Programa de Pós-Graduação/Departamento de Economia da Universidade Federal do Espírito Santo. E-mail: alhersco.vix@terra.com.br. Submetido: 16/abril/2013; Aprovado: 19/ setembro/2014.
} 


\section{INTRODUÇÃO}

Hoje, no debate econômico, tanto na sua dimensão teórica quanto empírica, tanto de um ponto de vista normativo quanto positivo, as referências à Economia Neoclássica são inevitáveis. Certas teorias, assim como suas aplicações em termos de políticas econômicas, reivindicam esta filiação; outras, ao contrário, se definem em oposição a esta matriz teórica (Dequech, 2007). Uma definição epistemológica da Economia Neoclássica tornou-se necessária, para poder definir a filiação teórica dos diferentes modelos propostos e para estudar o jogo de compatibilidades e de incompatibilidades entre as diferentes escolas de pensamento.

Contrariamente à análise de Kuhn (1991), a epistemologia de Lakatos permite explicar a coexistência de vários paradigmas, o que corresponde ao caso da Ciência Econômica. Lakatos reconhece que o progresso empírico não pode ser verificado imediatamente $(1978$, p. 49); assim, o caráter progressivo ou degenerativo do Programa de Pesquisa Científica (PPC) só será conhecido no longo prazo, após várias modificações das hipóteses auxiliares.

Após ter definido o conceito de implosão, o qual se relaciona diretamente com a abordagem de Lakatos, mostrarei como, e em que medida, a Nova Economia Institucional elaborada por Coase e Williamson, e a Nova Economia da Informação, oriunda dos trabalhos seminais de Akerlof, Grossman e Stiglitz, podem ser interpretadas como uma manifestação patente de implosão do PPC neoclássico ${ }^{1}$. Em última instância, o objetivo deste trabalho consiste em estudar até que ponto uma análise que se inspira no método preconizado por Lakatos permite explicar, a partir da história interna, a supremacia da Economia Neoclássica e, consequentemente, a partir de que ponto esta supremacia se explica por fatores que pertencem à história externa.

O texto está organizado como segue. Em uma primeira parte, definirei os instrumentos e o método de Lakatos e elaborarei uma análise lakatosiana do PPC neoclássico. Numa segunda parte, mostrarei como o surgimento e o desenvolvimento da Nova Economia Institucional e da Nova Economia da Informação provocaram a implosão do PPC neoclássico e permitem explicar seu caráter degenerativo.

\section{O PPC NEOCLÁSSICO: UMA ANÁLISE A PARTIR DAS CATEGORIAS DEFINIDAS POR LAKATOS}

\section{A epistemologia de Lakatos}

\section{Lakatos: a meio caminho entre Popper e Kuhn}

Para Lakatos (1970, p. 92), a reconstrução racional da História da Ciência só pode ser concebida a partir da história interna, sendo que "[...] external history is

\footnotetext{
${ }^{1}$ Seria possível tomar outros exemplos, como a teoria pós-keynesiana e a teoria do crescimento endógeno de Romer. Não obstante, no âmbito deste artigo, limitar-me-ei aos dois PPC mencionados.
} 
irrelevant for the understanding of science". A proposta de Lakatos consiste em definir o critério que permite, em relação a essas evoluções, escolher o PPC mais abrangente, o que implica que a Ciência seja concebida como um processo evolutivo e progressivo. Não obstante, surgem certas ambiguidades, no que diz respeito à hipótese do desenvolvimento autônomo da Ciência, em relação à história externa:

i) Um PPC é progressivo a partir do momento que permite prever fatos novos. Um PPC progressivo, no período $t$, pode se tornar degenerativo em $t+n$, à medida que os fatos novos a partir dos quais ele vai ser avaliado mudaram. Isso permite relativizar o conceito de progresso da Ciência e questionar, em última instância, sua autonomia. Esse progresso é relativo, pelo fato dele se relacionar com fatos que se modificam no decorrer do tempo: "We thus have a demarcation rule between science and non-science which is itself historical, involving the evolution of ideas over time as one of its necessary elements" (Blaug, 1976, p. 156). Essa relatividade histórica ressalta a diferença que existe entre as Ciências da natureza e as Ciências sociais (Hutchison, 2004, p. 183).

Outros elementos mencionados pelo próprio Lakatos limitam essa autonomia da Ciência: a respeito da definição do núcleo duro, Lakatos afirma que este é "[...] “irrefutable' by the methodological decision of its proponents" (1978, p. 50). Consequentemente, os critérios escolhidos para avaliar os PPC não são isentos de julgamentos de valor: "a rationality theory - or demarcation criterion - is to be rejected if it is inconsistent with an accepted 'basic value judgment' of the scientific elite" (Lakatos, 1970, p. 110). A história externa determina, pelos menos parcialmente, os julgamentos de valores adotados pela elite científica e os critérios de julgamento utilizados para aceitar e/ou rejeitar os diferentes PPC. No caso da comunidade científica não ser homogênea, haverá vários julgamentos de valores legítimos. Qual será, nesse caso, o critério adotado para aceitar, ou rejeitar, um determinado PPC? É possível falar em incomensurabilidade dos paradigmas, no sentido kuhniano?

ii) A posição de Lakatos se situa a meio caminho, entre aquela de Popper e de Kuhn. Em relação a Popper, o critério de demarcação contém uma dimensão histórica: a razão que permite escolher o PPC progressivo não é a- ou supra-histórica. Por outro lado, as escolhas feitas entre diferentes programas rivais não são unicamente determinadas por variáveis externas, conforme afirma Kuhn. Encontramos uma posição "mertoniana", na medida em que existem "external social factors that promote or impede the development of scientific knowledge, factors that can be studied by the sociology of science, but the objective content of scientific theories exists independently of these social factors" (Hands, 2004, p. 78). O conceito de implosão que desenvolverei aqui ressalta a não redutibilidade da Ciência às variáveis externas.

A problemática desenvolvida neste trabalho consiste em determinar, a partir de uma metodologia (pós-)lakatosiana, o peso respectivo das variáveis internas e externas: as variáveis externas começam a ser explicativas a partir do momento que as variáveis internas deixam de sê-lo. 


\section{A centralidade do conceito de Programa de Pesquisa Científico}

O conceito de PPC é o elemento central da démarche de Lakatos. Ele é composto por um núcleo central (hard core) e por uma heurística positiva. O núcleo central constitui a heurística negativa: esta é definida como "[...] paths of research to avoid" (Lakatos, 1978, p. 47), ou seja, como o que é incompatível com este núcleo central. Este é, por definição, irrefutável, e constitui os fundamentos do PPC; corresponde ao que é aceito pela comunidade científica. É em função desta definição que as hipóteses auxiliares protegem o núcleo duro.

A heurística positiva tem por função principal proteger o núcleo central, em relação às diferentes anomalias que o ameaçam. Ela permite evitar o modus tollens, ou seja, ela permite manter a integridade do núcleo duro. Sua função consiste em definir os problemas a serem estudados, prever fatos novos e integrar assim as diferentes anomalias (Lakatos, 1970, p. 99). Enquanto o núcleo duro é, por natureza, fixo, uma flexibilização das hipóteses auxiliares permite, simultaneamente, proteger este núcleo duro e prever novos fatos. O desenvolvimento e a modificação das hipóteses auxiliares têm que ser compatíveis com o núcleo duro do PPC: isto permite manter a coerência global do programa de pesquisa, intensificar seu desenvolvimento teórico, ou seja, sua capacidade de prever fatos novos, e evitar a armadilha do falseabilismo popperiano: "The positive heuristic of the programme saves the scientist from becoming confused by the ocean of anomalies" (Lakatos, 1978, p. 50).

Um dos objetivos principais da epistemologia de Lakatos consiste em construir um critério racional de escolha dos PPC e em elaborar assim uma reconstrução racional da História da Ciência. No âmbito de tal perspectiva, a História do Pensamento Econômico só pode ser concebida a partir de seus fundamentos epistemológicos, ou seja, a partir dessa reconstrução racional.

A tipologia estabelecida por Lakatos (1978, p. 99, 100 e 125) é a seguinte: um programa é teoricamente progressivo (theoretically progressive) se ele permite prever fatos novos; ele é empiricamente progressivo (empirically progressive) quando parte dessas previsões se realiza (Lakatos, 1978, p. 79). Um PPC é progressivo quando seu desenvolvimento teórico (theoretical growth) antecipa seu desenvolvimento empírico (empirical growth): o desenvolvimento das hipóteses auxiliares é tal que permite (a) prever e explicar novos fatos, essas hipóteses sendo compatíveis com o núcleo central, e (b) verificar, em parte, essas previsões a partir das observações empíricas. Um programa é estagnante quando seu desenvolvimento teórico ocorre depois de seu desenvolvimento empírico: ele se limita a fornecer explicações ad hoc.

Um PPC é um sistema estruturado e evolutivo: estruturado porque vários modelos podem se desenvolver a partir de uma base comum, o hard core (Hausman, 2004, p. 202). Evolutivo, a partir do momento que a reconstrução racional consiste em estudar a sucessão dos diferentes PPC, a partir de dois elementos: (a) o caráter progressivo (ou degenerativo) de um PPC é determinado de maneira relativa, a partir da comparação entre os diferentes PPC rivais, e (b) sua implosão, quando um determinado PPC desenvolve elementos que se tornam incompatíveis com al- 
guns componentes de seu núcleo duro. Nesse caso, conforme afirma Latsis (1976, p. 16), a respeito da heurística positiva, "[...] it cannot be given up without giving up the programme itself".

Essa implosão é um primeiro sinal de degenerescência do PPC original. À medida que o novo PPC se desenvolve a partir das "falhas" do antigo, é altamente provável que este primeiro seja progressivo, e que o antigo se torne degenerativo. A implosão surge quando a heurística positiva não permite mais compatibilizar a modificação das hipóteses auxiliares com os componentes do núcleo duro e, ao mesmo tempo, prever fatos novos. Essa démarche corresponde exatamente àquela descrita por Lakatos: um novo PPC pode ser inicialmente enxertado (grafted) sobre um programa mais velho: "As the young grafted programme strengthens, the peaceful co-existence comes to an end, the symbiosis becomes competitive and the champions of the new programme try to replace the old programme altogether" (Lakatos, 1978, p. 57).

Esse conceito de implosão permite realizar uma análise endógena da evolução dos diferentes PPC sem ter que recorrer à hipótese da autonomia da Ciência ou, ao contrário, da primazia das variáveis externas ao campo científico. Tal reconstrução racional constitui uma abordagem dinâmica (Latsis, 1976, pp. 15-6), permite explicar a coexistência de vários PPC em uma mesma época, e combina história interna e externa.

A dinâmica a partir da qual eu trabalhei é a seguinte: em um primeiro momento, a Economia da Informação e a Nova Economia Institucional nasceram no seio do PPC neoclássico. Esses modelos se desenvolveram e, a partir de um certo ponto, eles se tornaram incompatíveis com o núcleo duro do PPC neoclássico. Autores como Stiglitz, Coase e Williamson foram além de Stigler: a implosão desse PPC se manifesta pelo fato dos desenvolvimentos desses autores se tornarem incompatíveis com o núcleo duro. Além desse ponto, há uma autonomização dessas abordagens e a constituição progressiva de PPC concorrentes:

i) Stiglitz e Grossman mostraram que a presença de imperfeições da informação implica, obrigatoriamente, assimetrias da informação. Nesse caso, (a) o jogo de mercado não é mais Pareto eficiente, (b) os mercados não fecham em equilíbrio, mas aparecem racionamentos, (c) o sistema não converge para o equilíbrio.

ii) Coase ressalta o fato que a definição das externalidades entra em contradição com a teoria subjetiva do valor, e Williamson que o mercado não é, sistematicamente, a instância mais eficiente quando os ativos são específicos.

\section{O PPC NEOCLÁSSICO: DEFINIÇÃO EPISTEMOLÓGICA E CARACTERÍSTICAS}

\section{Algumas observações preliminares}

A dimensão normativa associada à Economia Neoclássica se explica e se justifica a partir dos dois Teoremas do Bem-Estar, os quais estabelecem uma relação 
de causalidade entre o Equilíbrio Geral (EG), as condições de Concorrência Pura e Perfeita (CPP) e a eficiência social avaliada a partir do critério de Pareto: "The two main theoretical results of the new welfare economics were the first and second fundamental theorems which linked the concept of a PO allocation to the Walrasian competitive equilibrium (CE)" (Hands, 2013, p. 4).

A eficiência social está diretamente ligada à concepção paretiana da justiça: à medida que o EG, nas condições de CPP, é automaticamente associado a um ótimo de Pareto, a partir do primeiro Teorema do Bem-Estar, e que a eficiência social é avaliada a partir deste ótimo, a construção neoclássica adquire uma dimensão normativa. Toda política econômica só pode ter como objetivo a preservação (ou a restauração) das condições de CPP, para o sistema alcançar automaticamente este ótimo social: "The efficient market has again gained ground as the only objective scientific reference for solving social problems. If an economic policy fails to work, this is attributed to the fact that it was not designed in accordance with market principles" (Burgenmeier, 1994, p. 351).

As diferentes escolas que pertencem ao PPC Neoclássico compartilham, no mesmo núcleo duro, o EG e o primeiro Teorema do Bem-Estar. Utilizando este arcabouço teórico, elas pretendem descrever assim a realidade e, simultaneamente, fornecer instrumentos normativos para elaborar as diferentes políticas econômicas (Stiglitz, 2003, p. 32; Blaug, 1976, p. 176)².

É interessante notar que a proposta epistemológica de Friedman refuta essa dimensão normativa: em seu artigo "The Methodology of Positive Economics" (2009), ele afirma que a economia tem que ser concebida como uma ciência positiva, e não normativa (op. cit., p. 4). A validade das hipóteses (ou teorias) não depende de seu realismo, mas de sua capacidade em elaborar previsões que se realizam com uma frequência "aceitável", à luz das "factual evidences" (idem, p. 9). Nos termos da epistemologia lakatosiana, essas hipóteses (ou teorias) se relacionam diretamente com a elaboração do cinturão protetor, ou seja, com a definição das hipóteses auxiliares, e não com o núcleo duro.

$\mathrm{Na}$ literatura atual, não encontramos uma definição essencialmente epistemológica do PPC neoclássico. Colander (2000) proclama "the death of neoclassical economics" e fala assim em hortodoxe economics, mainstream economics ou modern economics. Os argumentos são os seguintes: (a) autores que contestam o EG e seus principais resultados, fazem parte desta Economia Neoclássica, entre outros, Arrow, Akerlof, Stiglitz e Sen, "[...] but each operates outside the 'neoclassical framework' in portions of his work" (idem, p. 137), (b) a economia moderna consegue tratar da incerteza, dos equilíbrios múltiplos, da racionalidade limitada, e das imperfeições da informação, por exemplo, e (c) a teoria neoclássica é dominante pelo fato de não existir "[...] uma teoria alternativa com maior grau de falseabili-

\footnotetext{
${ }^{2}$ Existem outras definições possíveis da Economia Neoclássica (Weintraub, 1985, por exemplo). Não obstante, adotarei a definição de Stiglitz: ela permite definir com precisão o PPC neoclássica e, consequentemente, definir as incompatibilidades com os PPC concorrentes.
} 
dade capaz de explicar os fatos explicados pela teoria anterior e algum fato novo adicional" (Lisboa, 1998, p. 126).

O primeiro argumento é de ordem sociológica e não epistemológica; a esse respeito, Stiglitz (2003) deixa claro que ele propõe um paradigma alternativo em relação ao neoclássico. O terceiro argumento invoca uma argumentação popperiana e/ou lakatosiana para ressaltar a superioridade da economia da mainstream. A referência a Lakatos não é válida enquanto o núcleo duro e as hipóteses auxiliares do PPC examinado não forem definidos. O mesmo tipo de observação pode ser feito em relação ao segundo argumento.

É neoclássico quem se proclama neoclássico, sem referências a certos princípios metodológicos claramente definidos. Tal "definição" é puramente tautológica, e nega, de fato, a possibilidade de implementar uma análise epistemológica. Enquanto os diferentes componentes do PPC não forem definidos, não é possivel definir os limites deste PPC, nem elaborar uma reconstrução racional da Ciência Econômica a partir de sua história interna. Essa ausência de definição epistemológica permite ampliar o campo de investigação; no entanto, o que o PPC ganha em amplitude, ele perde em unidade metodológica e em poder normativo, na medida em que os diferentes modelos desenvolvidos não compartilham mais uma base comum, ou seja, um mesmo núcleo duro.

Apesar de Stiglitz não elaborar uma análise epistemológica explícita, seus trabalhos fornecem elementos que ressaltam a dimensão normativa do PPC neoclássico e que permitem definir seu núcleo duro e suas hipóteses auxiliares: os dois teoremas do Welfare associam o estado de CCP à eficiência paretiana (2003, p. 7) e formalizam assim a parábola da mão invisível smithiana. Se as condições de CPP se verificam, a alocação dos recursos é Pareto ótima, sob certas hipóteses. Isto implica, obrigatoriamente, que seja adotada a teoria subjetiva do valor, a partir de uma concepção ordinal. O equilíbrio geral é incompatível com a existência de incerteza, no sentido pós-keynesiano (Arrow, 1974a); os mercados são completos (Stiglitz, 1994, p. 29), o que é equivalente à ausência de incerteza, ou seja, à hipótese de ergodicidade.

Há uma série de condições a partir das quais este teorema funciona; essas condições compõem o cinturão protetor. A informação perfeita explica porque o conjunto dos mercados fecha em equilíbrio; da mesma maneira, não existe incerteza no que diz respeito à qualidade dos bens (a hipótese de homogeneidade), e os Direitos de Propriedade (DP) são plenamente eficientes e "gratuitos".

Conforme mostrarei na segunda parte deste trabalho, Grossman e Stiglitz $(1976,1980)$ vão mostrar que a modificação dessas hipóteses auxiliares é incompatível com a eficiência paretiana, ou seja, com um dos elementos do núcleo duro. Conforme Stiglitz afirma em vários trabalhos (notadamente Stiglitz, 1994, 2003), a Nova Economia da Informação produz esta implosão do programa neoclássico-padrão e, ao mesmo tempo, fornece elementos para construir um programa de pesquisa alternativo. 


\section{A estruturação do PPC neoclássico}

O núcleo duro é constituído por hipóteses irrefutáveis, por definição. Ele é composto pelos seguintes elementos:

- A teoria do valor utilidade subjetivo. De fato, não é possível "comprovar" esta hipótese; ela faz parte da própria construção do objeto de estudo e permite definir os problemas a serem estudados. As evidências fatuais, no sentido empregado por Friedman, serão definidas a partir desta teoria do valor.

- A hipótese de ergodicidade fornece os primeiros elementos que permitem entender como pode ser exercida a racionalidade individual. Os agentes maximizam suas funções de lucro e de utilidade pelo fato deles conhecerem as quantidades e os preços praticados nos mercados presentes e futuros.

O EG é incompatível com a existência de incerteza forte; "[...] uncertainties can tend to destroy markets, then we can conclude that the absence of some markets for future goods may cause others to fail " (Arrow, 1974a, p. 9). Nesse caso, não há mais market clearing no conjunto dos mercados, e, tendo em vista a interdependência generalizada dos mercados, os desequilíbrios se propagam para o conjunto dos mercados (Idem).

A teoria das expectativas racionais tem que ser concebida como uma outra representação desta racionalidade maximizadora: os agentes elaboram racionalmente suas expectativas, as probabilidades subjetivas convergem para o valor das probabilidades objetivas e, em média, eles não cometem erros de previsão.

- O ótimo de Pareto indica, para uma distribuição da renda dada e determinada exogenamente, qual é a situação que corresponde à maior eficiência social. Este conceito tem várias limitações (exploradas em Arrow, 1974b; Sen, 1982; Stiglitz 2003).

A construção do EG só faz sentido em função da definição da heurística positiva: os diferentes elementos da heurística positiva, notadamente o individualismo metodológico, a hipótese de homogeneidade e as outras condições de CPP, permitem "operacionalizar" o EG, associá-lo a um ótimo de Pareto e, no âmbito da uma abordagem normativa, estudar as diferentes imperfeições de mercado. Esta heurística positiva "defines problems, outlines the construction of a belt of auxiliary hypotheses, foresees anomalies and turns them victoriously into examples, all according to a preconceived plan" (Lakatos, 1970, p. 99). As aplicações em termos de políticas econômicas consistem em "flexibilizar" os diferentes mercados, no sentido de aproximá-los de um estado de CPP.

Passemos à definição das hipóteses auxiliares que, por definição, são refutáveis; a heurística positiva assegura a coerência global do PPC, ou seja, a compatibilidade entre a modificação dessas hipóteses e os elementos do núcleo duro (cf. quadro no Anexo).

\section{Individualismo metodológico e homogeneidade}

Uma preferência individual é qualificada de objetiva quando ela é exógena e quando ela não depende das preferências dos outros agentes (Orléan, 2011, p. 58). 
Esta hipótese é incompatível com a existência de externalidades, com comportamentos miméticos e com qualquer comportamento estratégico (A teoria dos jogos e do oligopólio, por exemplo). As relações entre os agentes são substituídas pelo leiloeiro (crieur de prix, na terminologia utilizada por Walras), o qual permite confrontar e compatibilizar as decisões de oferta e de demanda e assegurar assim o market clearing contínuo.

\section{Homogeneidade, preços, qualidade e equilíbrio}

A partir da análise de Lancaster (apud Orléan, 2011, pp. 61-2), as características dos bens são as mesmas para o conjunto dos consumidores. A qualidade é dada; cada consumidor escolherá uma cesta de bens, em função de suas preferências subjetivas. Existe assim uma dimensão objetiva na determinação da utilidade: as características, ou seja, as qualidades dos bens, são as mesmas para o conjunto dos consumidores. A dimensão subjetiva se explica pelo fato das escolhas dos consumidores serem diferentes, entre vários bens: “[...] de tal maneira que o elemento pessoal na escolha do consumo se relaciona com a escolha entre as características, e não com a alocação das características aos respectivos bens" (Lancaster, apud Orléan 2011, p. 62). Por hipótese, não há incerteza no que diz respeito à determinação da qualidade.

O sistema de preços fornece todas as informações necessárias relativas à qualidade dos bens e serviços: "[...] objects [...] cannot be distingued (before purchase) other than by price" (Stiglitz, 1987, p. 8): o preço depende diretamente da qualidade (Idem, p. 2). Conforme ressaltam Grossman e Stiglitz $(1976,1980)$ e Stiglitz (1987, 2011), o relaxamento dessa hipótese é incompatível com a hipótese dos mercados eficientes. A cada preço corresponde uma determinada qualidade, para o conjunto dos agentes. Há uma "objetivização" (Orléan, 2011, pp. 96, 97) da qualidade, e a homogeneidade se define a partir dessa relação unívoca entre preço e qualidade:

$$
\mathrm{p}=\phi(\mathrm{q})
$$

( $q$ representa a qualidade e $p$ o preço).

\section{O self enforcement}

O PPC neoclássico parte do pressuposto segundo o qual há self enforcement: de antemão, certas condições gerais essenciais são asseguradas, funcionam plenamente sem representar um custo privado nem social (Bowles e Gintis, 1993).

- Os custos de transação $(\mathrm{CT})$ relativos ao funcionamento do mercado e à implementação das trocas são, por princípio, nulos. Segundo Coase, essa hipótese corresponde, na Física, ao fato de considerar que os movimentos dos corpos se realizam sem atrito (1988).

A ausência de incerteza forte faz com que os contratos sejam, por natureza, 
completos, assim como os mercados. Consequentemente, os DP são plenamente eficientes, na medida em que os produtores controlam todas as modalidades de apropriação de sua produção. Esses DP são implementados sem custo nenhum. Eles permitem internalizar o conjunto das externalidades: a utilidade social coincide com a utilidade individual.

- O postulado de homogeneidade dos bens e dos serviços constitui um elemento essencial do PPC neoclássico: ele implica que o sistema de preços fornece gratuitamente as informações relativas à qualidade. Ao contrário, quando a economia é "relacional", a qualidade depende do comportamento dos agentes que participam da troca. Os contratos são, por definição, incompletos (Williamson, 2002) e os custos de transação obrigatoriamente positivos: eles consistem em monitorar o comportamento dos agentes oportunistas e em “conter" esta incerteza comportamental (Barzel, 1997).

\section{A IMPLOSÃO DO PPC NEOCLÁSSICO}

\section{As estratégias de imunização desenvolvidas pelo PPC neoclásico}

Weintraub considera que o núcleo duro do PPC neoclássico (que ele qualifica de neo-walrasiano) está sempre em construção, ou seja, não é fixo: "Consequently it must be the case that the hard core is not so fixed as a traditional Lakatosian appraisal may seem to suggest" (1985, p. 36). Não é mais possível definir o núcleo duro nem a heurística positiva; já que o núcleo duro é "flutuante", um determinado componente pode pertencer a este núcleo duro ou ao cinturão protetor. Backhouse (2004, p. 178) adota uma posição semelhante: "Lakatos's definition of a research programme in terms of an invariant hard core is too narrow". Trata-se de uma estratégia de imunização: quando uma anomalia ameaça o núcleo duro, uma modificação deste núcleo duro permite afastar essa ameaça.

Esse tipo de escolha confunde, propositadamente, modelos e PPC: um PPC se relaciona com os movimentos de longo prazo e com uma dinâmica interna que permite explicar suas diferentes evoluções. Representa a matriz comum (é o termo utilizado pelo próprio Kuhn) a partir da qual diferentes modelos são elaborados; um PPC permite desenvolver várias teorias e modelos. Por oposição, os modelos são elementos isolados, que se relacionam com uma lógica de "curto prazo", e que compartilham um mesmo núcleo comum. Em última instância, é impossível definir, a partir de critérios epistemológicos, um modelo como neoclássico sem definir, previamente, esta matriz original que ele compartilha com outros modelos. É preciso definir inicialmente os componentes do PPC neoclássico para estudar o jogo das compatibilidades e das incompatibilidades com outros PPC; por esta razão, estudarei agora os PPC ligadas à Nova Economia Institucional e à Nova Economia da Informação. 


\section{A Nova Economia institucional: da Economia do Bem-Estar à Economia dos Custos de Transação}

Para Pigou, externalidades positivas ou negativas surgem quando há uma divergência entre o produto marginal social e o privado (1920, cap. 2, seção 9). No caso mais específico da produção de externalidades negativas, o Estado, ou as outras autoridades públicas, têm que intervir para restabelecer as condições ótimas: "It is, however, possible for the State, if it so chooses, to remove the divergence in any field by 'extraordinary encouragements' or 'extraordinary restraints' upon investments in that field. The most obvious forms which these encouragements and restraints may assume are, of course, those of bounties and taxes" (Idem).

Adotaremos a definição de Hands (2013, p. 3): a Economia do Bem-Estar é a síntese entre a Economia de Pigou e de Marshall e o ótimo de Pareto. Se algum agente (ou grupo de agentes) for prejudicado pela existência de externalidades negativas, a situação deixa de ser Pareto ótima; o Estado tem que intervir, quer pela implementação de um regulamento, quer pela implementação de taxas e subsídios, para restabelecer este ótimo. Esta abordagem considera que a racionalidade dos agentes é substantiva, na medida em que eles podem avaliar sua utilidade (e desutilidade), por natureza, subjetiva. A Economia do Bem-Estar está diretamente ligada à teoria do valor utilidade subjetiva e ao ótimo de Pareto; as imperfeições implicam que certas hipóteses auxiliares sejam modificadas.

A análise de Coase é diferente; a reintrodução das funções específicas da firma, e de custos de transação positivos, faz surgir uma série de contradições. Edgeworth considerava implicitamente que os agentes alcançam uma situação ótima e que essa busca se implementa sem custos. Ao contrário, a partir do momento em que essa busca se traduz em custos de transação positivos, se esses custos são superiores à utilidade marginal, o sistema não alcança um ótimo de Pareto (Coase, 1988, pp. 184-5). Arrow já tinha percebido esse problema (1985, pp. 78-9) em relação ao ótimo de Pareto: ou as transações se efetuam fora da posição de equilíbrio, ou os agentes precisam de tempo para calcular e alcançar essa posição. Esse ajustamento progressivo rumo ao equilíbrio se traduz em custos e, consequentemente, perdas de bem-estar (Idem, p. 79).

Coase (1960) afirma que um Direito de Propriedade é definido a partir da utilização de um ativo determinado. Sua utilização implica que o detentor deste direito possa gerar externalidades que, obrigatoriamente, serão negativas para certos agentes, e apropriar-se do lucro gerado pela utilização do ativo. De um ponto de vista lógico, não é possível determinar uma externalidade se, antes, não forem determinados certos valores críticos que definem os limites desses direitos: o exemplo da determinação dos diferentes direitos de poluição ilustra perfeitamente esta situação. A definição desses valores críticos tem que ser socialmente aceita, ou seja, legitimada: a determinação desses valores críticos é institucional, e esses valores podem se modificar com o decorrer do tempo. Contrariamente à análise em termos de bem-estar social, há uma determinação social, e não subjetiva, das externalidades.

Esta concepção das externalidades torna obrigatória uma redefinição do cri- 
tério adotado para avaliar o bem-estar social. Isto constitui os fundamentos da crítica de Coase em relação à análise de Pigou. Coase adota um critério que ficou conhecido como o critério de Kaldor-Hicks (Pejovich, 1995), a partir do princípio seguinte: “[...] pode ser que o valor da produção seja mais elevado se aqueles que causaram a externalidade negativa não têm obrigação de indenizar aqueles que foram prejudicados" (Coase, 1988, p. 204). Coase vai avaliar o bem-estar a partir da produção total realizada; nesse caso, a compensação dos poluídos não tem que ser sistematicamente realizada. A solução mais eficiente será aquela que maximiza a produção e minimiza os CT; à medida que esta compensação não for efetuada, a situação deixa de ser Pareto ótima e, em todos os casos, corresponde a um second best (McCloskey, 1998, p. 368).

Stigler (1966) tenta integrar a análise de Coase no PPC neoclássico, a partir da formulação do famoso "Teorema de Coase". Partindo do princípio segundo o qual os CT são nulos, a racionalidade dos agentes substantiva, os contratos completos e os DP totalmente definidos, o nível da produção total não depende da distribuição inicial dos DP, e a negociação privada dos DP é sempre mais eficiente que uma regulação pigouviana. O próprio Coase refuta esta interpretação (1988, pp. $7,32,50)$, e isso por duas razões: primeiramente, não faz sentido considerar CT nulos, tendo em vista que a contribuição de Coase consiste justamente em analisar uma economia “concreta”, na qual os CT são, por natureza, positivos. No seu artigo de 1960, Coase relaxa logo a hipótese de CT nulos. Segundo, Coase não demonstra que o mercado, ou a negociação privada, constitui, sistematicamente, a solução mais eficiente: "It is clear that an alternative form of economic organization which could achieve the same result that less cost than would be incurred by using the market would enable the value of production to be raised" $(1960$, p. 8).

Para compatibilizar uma análise em termos de CT e de DP com alguns componentes do PPC neoclássico, Stigler tem que supor que os DP sejam plenamente definidos e que os contratos sejam completos: ele tem que supor a ausência de incerteza. No seu artigo de 1937, Coase já afirmava que a existência da firma se justifica a partir da incerteza, e que, em determinados mercados, notadamente no mercado do trabalho, os contratos são, por natureza, incompletos (p. 392).

Em todos esses casos, contrariamente à análise em termos de bem-estar, a flexibilização das hipóteses auxiliares torna-se incompativel com dois componentes do núcleo duro: a teoria do valor subjetiva e o ótimo de Pareto. Na medida em que a determinação das externalidades é institucional, essas não dependem da avaliação subjetiva dos consumidores/usuários.

\section{A Nova Economia da Informação}

Uma outra ruptura epistemológica a partir da qual se desenvolve o mecanismo de implosão aparece com os trabalhos de Salop e se torna efetiva com os trabalhos de Akerlof e de Stiglitz. A vertente neoclássica da Economia da Informação parte dos seguintes pressupostos (Stigler, 1961): não existe incerteza, na medida em que os consumidores igualam o custo da informação com sua utilidade marginal ( $\mathrm{p}$. 
216), e os produtos são, por hipótese, homogêneos (p. 222). Se os primeiros trabalhos elaborados no âmbito da teoria neoclássica podem ser considerados seminais (Stigler, p. 961), seus limites explicativos logo apareceram. Provém do fato de que os principais postulados da Economia Neoclássica foram mantidos: a ausência de incerteza e a homogeneidade dos bens e dos serviços. Isso permite manter as preferências individuais fixas e objetivas, e ignorar assim as assimetrias da informação ligadas ao risco moral e às suas implicações no que diz respeito à qualidade dos bens e dos serviços. Se o PPC neoclássico tem condições de estudar as imperfeições da informação, ele não pode considerar as assimetrias de informação: estas são incompatíveis com o ótimo de Pareto.

$\mathrm{Na}$ sua análise dos carros usados (lemon market), Akerlof (1970) refuta a hipótese de homogeneidade dos bens: na medida em que existem dois tipos de vendedores, os honestos e os desonestos, a um mesmo preço correspondem várias qualidades. Os comportamentos oportunistas e as externalidades positivas e negativas são analisados no que diz respeito à oferta:

$$
\mathrm{q}_{1}, \mathrm{q}_{2}, \ldots, \mathrm{q}_{\mathrm{n}}=\phi(\mathrm{p})
$$

Mantendo a hipótese segundo a qual a qualidade dos bens é constante, trabalhos posteriores (Salop, 1976 Stiglitz, 1994, p. 42) mostraram que a dispersão dos preços gera imperfeições da informação: nesse caso, há um custo associado à informação. Os consumidores vão comparar o custo da informação (assimilado a um custo marginal) com a possibilidade de obter o mesmo bem por um preço inferior (a utilidade marginal): se esse custo for maior que a diferença de preços, o consumidor não vai comprar essa informação. Haverá assim vários preços correspondendo a uma mesma qualidade: a dispersão dos preços e as imperfeições que ela gera fazem com que um mesmo bem possa ser vendido com preços diferentes. Isso é contrário à lei do preço único:

$$
\mathrm{p}_{1}, \mathrm{p}_{2}, \ldots \mathrm{p}_{\mathrm{n}}=\phi(\mathrm{q})
$$

Os trabalhos de Stiglitz e Grossman $(1976,1980)$ e Stiglitz (1987) aprofundam o estudo dos mecanismos decorrentes da existência de imperfeições da informação, privilegiando os componentes da demanda:

(a) A refutação da hipótese dos mercados eficientes foi inicialmente realizada por Grossman e Stiglitz (1976), e pode ser resumida a partir das seguintes relações:

$$
\begin{aligned}
& \mathrm{E}_{1}=\mathrm{U}_{1}-\mathrm{p}_{1}-\mathrm{c}_{1} \\
& \mathrm{E}_{2}=\mathrm{U}_{2}-\mathrm{p}_{2}-\mathrm{c}_{2}
\end{aligned}
$$

E representa o excedente líquido (esperado) do consumidor, $\mathrm{U}$ a utilidade total esperada, $p$ e $c$ respectivamente, o preço dos bens e serviços e o custo pago pela informação. $\mathrm{O}$ índice 1 se relaciona com os consumidores pouco informados, $\mathrm{O}$ índice 2 com os informados.

Este mecanismo pode ser interpretado da seguinte maneira: no equilíbrio, $\mathrm{E}_{1}$ $=\mathrm{E}_{2}$, ou seja, $\mathrm{U}_{1}-\left(\mathrm{p}_{1}+\mathrm{c}_{1}\right)=\mathrm{U}_{2}-\left(\mathrm{p}_{2}+\mathrm{c}_{2}\right)$. Para $\mathrm{c}_{2}$ e $\mathrm{c}_{1}$ superiores a 0 , não há equi- 
líbrio concorrencial pelo fato de não existir um preço único ${ }^{3}$. No equilíbrio concorrencial, o excedente esperado por parte dos consumidores pouco informados é igual ao excedente esperado por parte dos consumidores informados; $\mathrm{U}_{2}-\mathrm{U}_{1}$ é compensada por $\mathrm{c}_{2}-\mathrm{c}_{1}$. Os consumidores não informados se beneficiam das externalidades positivas produzidas pelos consumidores informados e o equilíbrio é concorrencial.

O paradoxo da hipótese dos mercados eficientes pode ser enunciado da seguinte maneira: para os mercados serem eficientes, o sistema de preços tem que divulgar gratuitamente a informação dos agentes informados para os agentes não informados. Mas, neste caso, ex post, o excedente líquido dos agentes informados é igual àquele dos agentes não informados. Nesse caso, a eficiência dos mercados, e o ótimo de Pareto que lhe corresponde, são incompatíveis com a hipótese da racionalidade microeconômica: por que os agentes vão querer comprar informações se o sistema de preços divulga gratuitamente as informações qualitativas para os agentes não informados?

A solução proposta por Stiglitz e Grossman consiste em afirmar que o excedente líquido dos agentes informados é maior que aquele dos agentes não informados; esta solução pode ser formalizada da seguinte maneira:

$$
\begin{aligned}
& \mathrm{E}_{2}=\mathrm{U}_{2}-\left(\mathrm{p}_{2}+\mathrm{c}_{2}\right)>\mathrm{E}_{1}=\mathrm{U}_{1}-\left(\mathrm{p}_{1}+\mathrm{c}_{1}\right) \\
& \text { com } \mathrm{p}_{1}>\mathrm{p}_{2}, \mathrm{c}_{2}>\mathrm{c}_{1} \text { e } \mathrm{U}_{2}>\mathrm{U}_{1}
\end{aligned}
$$

À renda informacional corresponde uma renda extra. Esta situação é incompatível com o ótimo de Pareto, pelo fato do ganho de utilidade por parte dos agentes informados corresponder à perda de utilidade por parte dos agentes não informados: a modificação das hipóteses auxiliares produz resultados incompatíveis com o núcleo duro.

Os trabalhos posteriores de Stiglitz (por exemplo, 1987) aprofundaram este tipo de abordagem, mostrando que a qualidade do bem ou do serviço depende diretamente do preço; este tipo de análise é geralmente aplicado no caso do mercado do trabalho e no mercado de crédito. Temos a seguinte relação:

$$
\mathrm{q}=\phi 2(\mathrm{p}), \operatorname{com} \phi 2^{\prime}>0
$$

Nesse caso, há generalização dos racionamentos nos diferentes mercados e afastamento em relação à posição de equilíbrio. Em uma situação inicial de desemprego, a oferta de trabalho é superior à demanda: a solução neoclássica consiste em uma diminuição dos preços reais do trabalho para restabelecer o equilíbrio e alcançar a situação de pleno emprego. O conceito de salário de eficiência mostra claramente que a qualidade do trabalho (sua produtividade efetiva) depende diretamente de seu preço, e que a demanda de trabalho depende desta mesma qualidade. Em uma situação inicial de desemprego, a queda do preço do trabalho se traduz

\footnotetext{
${ }^{3}$ Stiglitz (2003, p. 13) considera que um dos princípios da Economia Neoclássica é "the law of the single price".
} 
por uma diminuição da qualidade desse trabalho e, consequentemente, por uma queda da demanda; está havendo uma ampliação do desequilíbrio inicial e a hipótese de market clearing não é mais verificada. A flexibilidade dos salários não permite a volta para a posição de equilíbrio. Isso é incompatível com os principais resultados do EG, ou seja, com a convergência para a posição de equilíbrio, e com o fato dessa posição caracterizar-se por market-clearing.

\section{Da implosão ao caráter degenerativo}

É importante entender por que a implosão leva à degenerescência do PPC de origem: novos PPC se desenvolvem, inicialmente, no seio do PPC dominante, para explicar certas anomalias. Essa implosão nasce das "insuficiências" do PPC de origem no que diz respeito à explicação de fatos novos e à previsão desses fatos. $A$ implosão representa o primeiro momento da degenerescência do PPC de origem.

No que diz respeito à Nova Economia da Informação, as diferentes concepções relativas à natureza do salário de eficiência são características da diferença entre os dois PPC. Para a Economia Neoclássica, é a rigidez dos salários reais que explica o desemprego. Nesse caso, o desenvolvimento teórico vem depois do desenvolvimento empírico: os economistas neoclássicos têm que explicar ex post um fato que não foi previsto. Ao contrário, a Nova Economia da Informação mostra que são as assimetrias de informação entre empregados e empregadores que produzem este racionamento; o modelo teórico antecipa os fatos e permite prevê-los. Esse tipo de observações pode ser estendido ao conjunto dos mercados, a partir do momento em que a hipótese de homogeneidade for abandonada e que aparecerem assimetrias entre os diferentes agentes que participam da troca: mercado dos bens e dos serviços e mercado do crédito bancário, por exemplo (Stiglitz, 1987).

O mesmo tipo de observações e de conclusões se aplica à comparação entre a Economia Neoclássica e a Nova Economia Institucional. CT positivos são a consequência de uma modificação da natureza econômica dos bens; à medida que esses bens se tornam específicos, no sentido definido por Williamson (2002), os DP não podem mais ser plenamente definidos nem totalmente eficientes, as imperfeições da informação implicam assimetrias, as quais permitem o desenvolvimento de comportamentos oportunistas. Aqui, também, a situação deixa de ser Pareto ótima.

Há vários pontos comuns entre a Nova Economia Institucional e a Nova Economia da Informação. A respeito das assimetrias de informação Stiglitz (2000, p. 1444) afirma que essas "[...] imply that markets and contracts cannot be complete." e que "Transaction costs (Williamson 1979) provide the major alternative explanation for incomplete contracts [...]". Enquanto esses dois PPC fornecem explicações ex ante, o PPC neoclássica só fornece explicações ex post, a partir das tentativas de integração dessas imperfeições. Lembre-se, a título de exemplo, a tentativa malsucedida de Stigler de integrar a análise de Coase no programa neoclássico.

Em síntese, é possível afirmar que o PPC neoclássico se limita a modificar, ex post, suas hipóteses auxiliares, para poder explicar esses fatos novos: rigidez dos 
salários e dos preços, não neutralidade da moeda no curto prazo, não convergência para o steady-state e imperfeições de informação (Arrow, 1974a).

\section{CONCLUSÃO}

O caráter degenerativo ou estagnante do PPC neoclássico aparece em dois níveis: além de fornecer apenas explicações ad hoc de fatos novos, a modificação das hipóteses auxiliares (necessárias para explicar esses fatos novos) produz resultados incompatíveis com o núcleo duro. A implosão desse programa constitui a primeira fase de um processo degenerativo. O excesso de formalização matemática e a ausência de uma definição epistemológica desse $\mathrm{PPC}^{4}$ não conseguem mais ocultar suas "falhas", nem a fase de implosão na qual ele se encontra, hoje. A Nova Economia Institucional e a Nova Economia da Informação constituem PPC alternativos que, no âmbito dessa comparação, podem ser qualificados de teoricamente progressivos.

Por que o PPC neoclássico continua sendo dominante, nas instituições de ensino, nos cursos universitários e como referencial em relação a boa parte das políticas econômicas implementadas, hoje? A análise epistemológica, e mais especificamente lakatosiana, não explica esse fato. A resposta tem que ser buscada na história externa; ela se relaciona diretamente com uma análise sociológica do campo social constituído pela pesquisa e pelo ensino da Economia.

Não obstante, a análise epistemológica permite definir o peso relativo do argumento sociológico para explicar esse efeito de dominação. Este é devido à história externa, e à influência das variáveis sociais e históricas. Esta análise sociológica deve amplamente utilizar os trabalhos de Bourdieu (1984) e de Sarte (1986) para explicitar a natureza desses determinismos externos ao campo científico; o conceito sartriano de projeto (Sartre, 1986, p. 56) ressalta as complementaridades entre os determinismos externos e internos, e as mediações necessárias operadas pela estrutura do campo em relação às variáveis externas. Cabe ao sociólogo implementar uma análise desse tipo.

\section{REFERÊNCIAS BIBLIOGRÁFICAS}

Akerlof, G. (1970), “The market for "lemons": Qualitative uncertainty and the market mechanism”, Quartely Journal of Economics, Aug. 1970, 89, pp. 488-500.

Arrow, Kenneth J., (1974a), "Limited knowledge and economic analysis", The American Economic Review, Marh 1974, pp. 1-10.

Arrow, Kenneth J. (1974b), "General economic equilibrium: purpose, analytic techniques, collective choice”, The American Economic Review, Vol. 64, No. 3, pp. 253-272.

\footnotetext{
${ }^{4}$ Hodgson (2007) formula conclusões parecidas quando discute o formalismo (p. 13) e as fraturas que apareceram no edifício de EG (p. 7).
} 
Arrow, Kenneth J. (1985), “The potential and limits of the market resource allocation”, in G.R. Feiwel (ed.), Issues in Contemporary Microeconomics and Welfare, London, Macmillan, pp. 107-124.

Backhouse, Roger E. (2004), "Introduction”, New Directions in Economic Methodology, Edited by Roger E.Backhouse, Taylor \& Francis e-Library, New York, Routledge, 1-26.

Barzel, Yoram (1997), Economic Analysis of Property Rights, Cambridge University Press.

Blaug, Mark (1976), "Kuhn versus Lakatos or paradigms versus research programmes in the history of economics", in Method and Appraisal in Economics, edited by Spiro J. Latsis, Cambridge University Press, pp. 149-198.

Bourdieu, Pierre (1984), Questions de Sociologie, Paris: Les Editions de Minuit.

Bowles Samuel and Gintis Herbert (1993), "The revenge of homo economicus: contested exchange and the revival of political economy", The Journal of Economic Perspectives, 7 (1), pp. 83-102.

Burgenmeier B. (1994), “The misperception of Walras”, The American Economic Review, Vol. 84, No. 1, pp. 342-352.

Coase, R. H. (1937), “The nature of the firm”, Economica, New Series, v. 4, n. 16. pp. 386-405.

Coase, R. H. (1960), “The problem of social cost”, Journal of Law and Economics, 3, pp. 1-43.

Coase, R. H. (1988), The Firm, the Market and the Law, Chicago, University of Chicago Press.

Colander David (2000), "The death of neoclassical economics", Journal of the History of Economic Thought, Vol. 22, No. 2, pp. 127-143.

Dequech, David (2007), "Neoclassical, mainstream, orthodox and heterodox economics", Journal of Post Keynesian Economics, vol.30.2, 365-378.

Friedman Milton (2009), The Methodology of Positive Economic, Cambridge, Cambridge University Press.

Grossman S.J. and Stiglitz J.E. (1976), "Information and competitive price system", The American Economic Review, Vol. 66 no. 2, pp. 246-253.

Grossman S.J. and Stiglitz J.E. (1980), "On the impossibility of informationally efficient markets", The American Economic Review, Vol. 70, No. 3, pp. 393-408.

Hands, D. Wade (2004), “The sociology of scientific knowledge: some thought on the possibilities", in New Directions in Economic Methodology, Edited by Roger E.Backhouse, Taylor \& Francis e-Library, New York, Routledge, pp. 76-110.

Hands, D. Wade (2013), “Mark Blaug on the normativity of welfare economics” Erasmus Journal for Philosophy and Economics, Volume 6, Issue 3 (Special Issue), pp. 1-25.

Hausman, Daniel M. (2004), "Kuhn, Lakatos and the character of Economics", in Blackhouse, Roger E., edited (2004) New Directions in Eonomic Methodology. New York: Routledge: 195-215.

Hodgson, Geoffrey M., (2007) "Evolutionary and institutional economics as the new mainstream?". Evolutionary and Institutional Economics Review, 4(1), September 2007, pp. 7-25.

Hitchison, T.W. (2004) "Ends and means in the methodology of Economics", in Blackhouse, Roger E., edited (2004) New Directions in Eonomic Methodology. New York: Routledge: 27-34.

Kuhn, Thomas S. (1991[1962]), A Estrutura das Revoluções Científicas, São Paulo, Editora Perspectiva.

Latsis Spiro J. (1976), “A research programme in economics” Method and Appraisal in Economics, op. cit., $1-42$.

Lakatos, Imre (1970), "History of science and its rational reconstructions", Proceedings of the Biennial Meeting of the Philosophy of Science Association, pp. 91-136.

Lakatos, Imre (1978), "The methodology of scientific research programs", Philosophical Papers Volume I Edited by John Worral and Gregory Currie, Cambridge University Press.

Lisboa, Marcos (1988), "A miséria da crítica heterodoxa”, Revista de Economia Contemporânea n.3.

McCloskey, Deirde (1998), “The so-called Coase theorem”. Eastern Economic Journal, v. 24, n. 3, pp. 367-371.

Orléan, André (2011), L’Empire de la Valeur. Refonder l'Économie, Paris, Éditions du Seuil.

Pejovich, S. (1995), "Economic analysis of institutions and systems". Kluwer Academic Publishers, International Studies in Economics and Econometrics, v. 33. London, Dordrecht-Boston.

Pigou A.C. (1920), The Economics of Welfare, London, MacMillan and Co. 
Possas, Mário (1995), “A cheia do main stream. Comentários sobre os rumos da Ciência Econômica”, Texto para discussão, Rio de Janeiro, UFRJ/IEI.

Salop Steve (1976), "Information and monopolistic competition", The American Economic Review, Vol. 66, No. 2, Papers and Proceedings of the Eighty-eighth Annual Meeting of the American Economic Association (May, 1976), pp. 240-245.

Sartre, Jean-Paul (1986), Questions de Méthode, Paris, Editions Gallimard.

Sen, Amartya (1982), Choice, Welfare and Measurement, Oxford, Blackwell.

Stigler, George J. (1966), The Theory of Price. 3.ed., New York, Macmillan.

Stigler, George J. (1961), The economics of information”. The Journal of Political Economy, Vol. 69, No. 3, pp. 213-225.

Stiglitz Joseph E. (1987), “The causes and consequences of the dependence of quality on price”, Journal of Economic Literature, vol. XXV, pp. 1-48.

Stiglitz Joseph E. (1994), Whiter Socialism?, Massachusetts, MIT.

Stiglitz Joseph E. (2000), "The contribution to the economics of information to twentieth century economics”, The Quarterly Journal of Economics, November.

Stiglitz Joseph E. (2003), "Information and the change in the paradigm in economics, Part 1", American Economist, pp. 6-26.

Stiglitz Joseph E. (2011), "Rethinking macroeconomics: what failed and how to repair it", Journal of the European Economic Association, pp. 591-645.

Weintraub, R. (1985), “Appraising general equilibrium analysis”, Economics and Philosophy, Vol. 1, 1985, pp. 23-37.

Williamson Oliver (2002), “The theory of the firm as governance structure: from choice to contract Journal of Economic Perspectives, v. 16, n. 3, pp. 171-195.

ANEXO - A implosão do PPC neoclássico

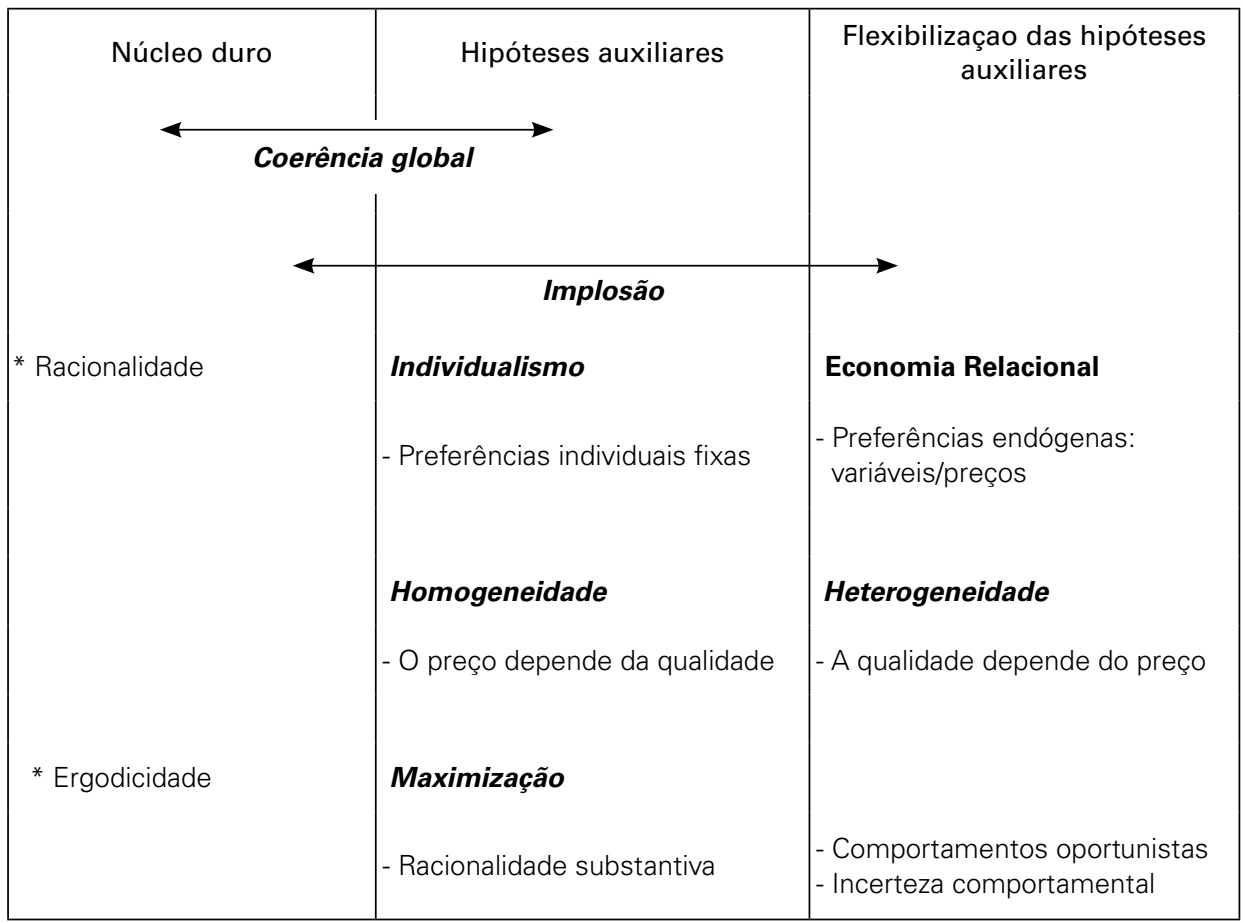




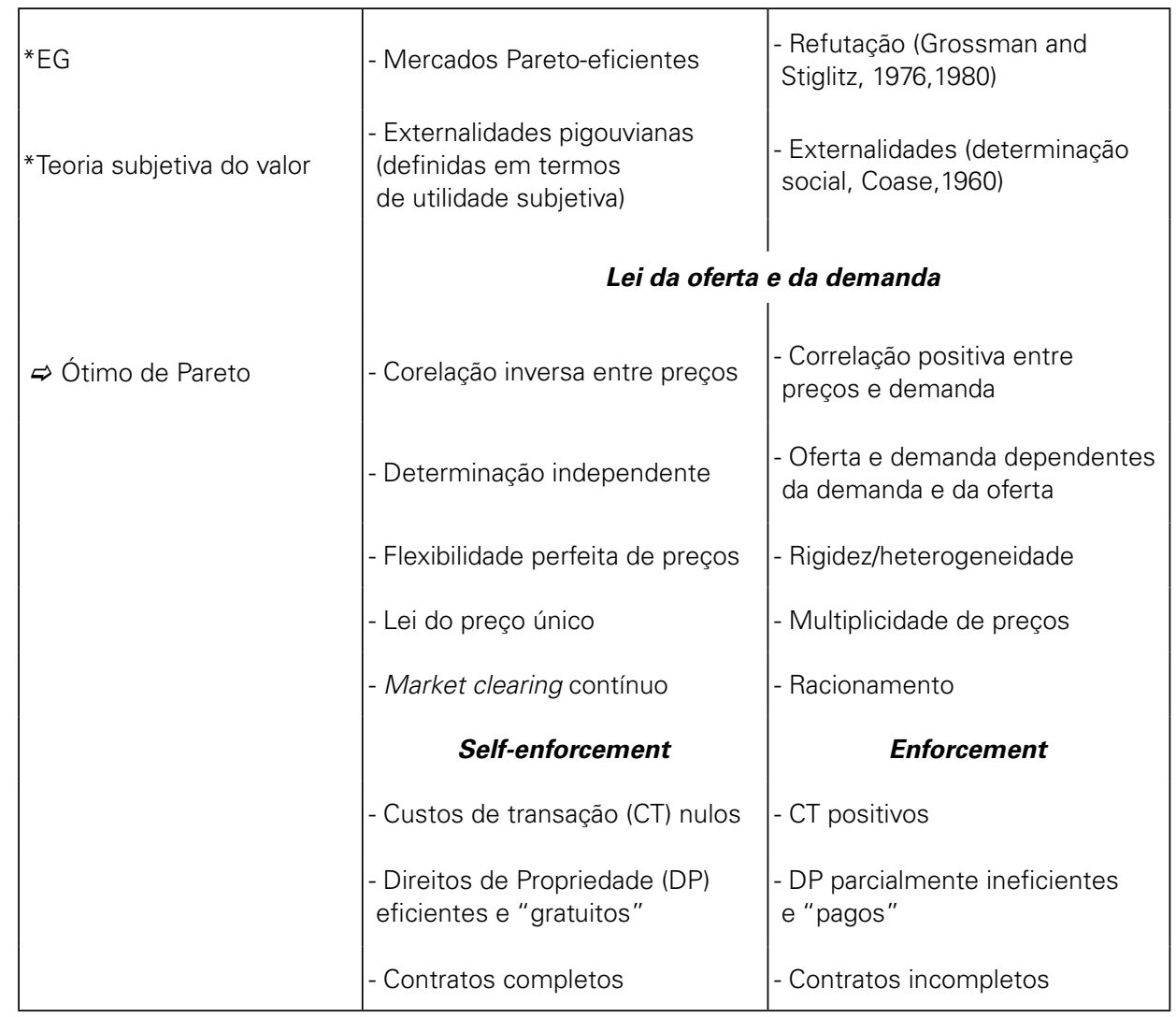

